\title{
The future of achalasia therapy: expanding the minimally invasive armamentarium and risk of secondary gastroesophageal reflux
}

\author{
Kelsey F. Angell, Jeffrey M. Marks \\ Department of Surgery, University Hospitals Cleveland Medical Center and Case Western Reserve University School of Medicine, Cleveland, Ohio, USA \\ Correspondence to: Jeffrey M. Marks, MD, FACS, FASGE. Department of Surgery, University Hospitals Cleveland Medical Center, 11100 Euclid \\ Avenue, Cleveland, Ohio 44106-5047, USA. Email: jeffrey.marks@uhhospitals.org. \\ Provenance: This is an invited Editorial commissioned by Section Editor Dr. Di Lu (Department of Thoracic Oncology, Nanfang Hospital, Southern \\ Medical University, Guangdong, China). \\ Comment on: de Pascale S, Repici A, Puccetti F, et al. Peroral endoscopic myotomy versus surgical myotomy for primary achalasia: single-center, \\ retrospective analysis of 74 patients. Dis Esophagus 2017;30:1-7.
}

Submitted Sep 14, 2017. Accepted for publication Sep 18, 2017.

doi: $10.21037 /$ jtd.2017.09.127

View this article at: http://dx.doi.org/10.21037/jtd.2017.09.127

Achalasia is a primary motor disorder of the esophagus characterized by insufficient lower esophageal sphincter (LES) relaxation and loss of esophageal peristalsis (1). Patients often present with progressive dysphagia, regurgitation, chest pain and weight loss. Manometry studies showing an absence of esophageal motility and incomplete LES relaxation without evidence of mechanical obstruction can confirm the diagnosis of achalasia (2). Standard of care options for therapy previously included surgical myotomy, including laparoscopic Heller myotomy (LHM) and endoscopic pneumatic dilation (PD) (3). Studies have demonstrated more durable symptom improvement with LHM compared with PD $(4,5)$. However, in a group of 73 patients treated with LHM, excellent or good responses were reported in $89 \%$ of patients at 6 months and $57 \%$ of patients at 6 years of follow-up, demonstrating decreasing efficacy of LHM with time (6). A novel alternative to LHM or PD, per-oral endoscopic myotomy (POEM) was first reported in 2008 by Inoue as a minimally invasive therapy for achalasia (7). The first POEM was performed in the United States in 2010 at the Oregon Clinic and has emerged as an endoscopic alternative to surgical myotomy. Early studies have shown that POEM is as safe and effective as surgical myotomy, with shorter procedure and recovery times and fewer serious complications (8).

Pascale et al. recently reported the outcomes of 74 patients who underwent surgical or endoscopic myotomy for achalasia over a 3 -year period at a single institution.
This retrospective review included patients with symptomatic achalasia and a baseline Eckardt score $>3$, with diagnosis confirmed by barium swallow, manometry and esophagogastroduodenoscopy. Thirty-two patients underwent POEM and 42 patients underwent surgical laparoscopic myotomy with Dor fundoplication (SM). Procedures were performed by a single endoscopist or one of two surgeons. The two patient groups were similar in characteristics including age, sex, symptoms, duration of symptoms and prior therapies for achalasia. Over $96 \%$ of patients in each group had type II achalasia. Operative time for POEM was shorter than SM (63 vs. 76 minutes, $\mathrm{P}=0.00005)$, and the myotomy was longer in POEM group (12 vs. $9 \mathrm{~cm}, \mathrm{P}=0.00001$ ). The POEM group had no postoperative complications, while the SM group had two $(4.7 \%)$ postoperative complications, including one patient who required repeat laparoscopy for port site bleeding. Median postoperative stay was slightly longer for the SM group at $3 v s .2$ days for the POEM group $(\mathrm{P}=0.0014)$. Median Eckardt scores decreased from 6 to 1 in both groups. The most frequent intraoperative complication in the POEM group was pneumoperitoneum in $9.3 \%$ of patients requiring decompression at the end of the procedure, while $11.9 \%$ of patients undergoing SM developed intraoperative mucosal tears which had no overall clinical impact on the patient's care.

This study supports prior literature reporting POEM as a safe, effective and minimally invasive treatment for 
achalasia. POEM has been reported to have shorter operative times and less blood loss than laparoscopic myotomy, with no difference in complication rate or length of stay (9). Additionally, POEM offered a longer myotomy than SM, which may allow more successful treatment and symptom reduction for patients with type III achalasia or general myotomy length customization. A recent report at SAGES provided 5-year follow up data for a series of 29 patients who had undergone POEM, evaluating symptoms scores and need for recurrent intervention (10). Twenty-three patients underwent POEM for achalasia, and 19 of those 23 patients had Eckardt scores of 3 or less at 5 years post-procedure. This longer term data supports the durable results of POEM for achalasia therapy. Authors noted this series included some of the first patients to undergo POEM in the United States and results may reflect early learning curves of surgeons performing the procedure. As surgeons gain additional experience performing these procedures and managing patients post-POEM, we may see improved clinical outcomes for patients. Longer term follow up for patients undergoing the procedure more recently may demonstrate higher rates of symptom score reduction, shorter hospital stays and overall lower complication rates.

The authors also reported an increased postoperative incidence of gastroesophageal reflux (GERD) in the POEM group, which is in accordance with reported GERD rates in other POEM series. Over half of the patients underwent evaluation for GERD at follow up, with $40 \%$ of the POEM group and $5 \%$ of the SM group with documented evidence of endoscopic esophagitis. Five patients in the POEM group and four in the SM group had pathologic DeMeester scores at follow up. Of the POEM patients with endoscopic evidence of esophagitis, 4 had LA grade A, 3 had LA grade $\mathrm{B}$ and 1 had findings consistent with LA Grade D. The SM group had one patient with LA grade A esophagitis. Five (28\%) of patients in the POEM group had pathological DeMeester scores, compared with $4(22 \%)$ patients in the SM group. Swanstrom et al. reported a series of eighteen patients who underwent POEM for achalasia, with all patients reporting dysphagia relief, but $46 \%$ of patients had objective evidence of GERD at 6-month $\mathrm{pH}$ studies (8). In a review of 500 consecutive POEM performed over a 5 years, 268 patients had esophagitis on endoscopy (64.7\%), but only $71(16.8 \%)$ had reflux symptoms. At $1-2$ years follow up, 9 of 16 patients had findings of esophagitis on endoscopy, while only $19.4 \%$ had heartburn symptoms. At 3-year follow up, there was no change in the rate of esophagitis on endoscopy, but $56.3 \%$ of patients had heartburn symptoms (11). In another analysis of 100 consecutive patients undergoing POEM over a 3-year period, $38 \%$ of patients had evidence of abnormal acid exposure on postoperative testing while only 14 patients were asymptomatic and no patients required additional antireflux procedure (12).

This provokes discussion over the need for an antireflux procedure in the POEM group, either as a standard of care at time of POEM or after additional follow up and evaluation for reflux. The long term consequences of untreated GERD in patients after POEM are not well studied and the follow up data on GERD development in SM is limited as well. GERD after all types of myotomy is fairly common, however adding an antireflux procedure such as fundoplication to decrease GERD may also increase postoperative dysphagia. POEM is performed without disruption of the ligaments around the esophagus, which would theoretically make these patients less at risk for developing postoperative GERD. In a series of 105 patients undergoing POEM at a single center, 70 were followed up three months after the procedure with Eckardt scores, manometry, endoscopy and $\mathrm{pH}$ monitoring. LES pressures and Eckardt scores all showed significant reductions, while 5 patients developed symptomatic GERD and 11 developed moderate reflux esophagitis, all of which were controlled with proton pump inhibitor therapy (13). Frequencies of GERD after surgical myotomy without fundoplication are similar after thoracotomy, laparotomy, thoracoscopy, and laparoscopy, ranging from $28 \%$ to $31 \%$ (4). Adding fundoplication as an antireflux measure after myotomy has been shown to decrease the risk of GERD to $14 \%, 8 \%$ and $9 \%$ after thoracotomy, laparotomy, and laparoscopy (4). Partial fundoplication has a lower dysphagia rate at $2.8 \%$ compared with $15 \%$ with total fundoplication at 5 -year follow (14). A multicenter, randomized controlled trial showed no differences in dysphagia after anterior (Dor) or posterior (Toupet) fundoplications, but the Dor fundoplication group had a statistically insignificant higher rate of abnormal $\mathrm{pH}$ studies at $41.7 \%$ versus $21 \%$ (15). These rates demonstrate that GERD is a problem after LHM even with partial fundoplication as an antireflux measure. A large meta-analysis of 468 patients with achalasia found lower rates of GERD in patients who underwent LHM versus POEM. However, the included studies over a 10-year period through 2015 were all limited to follow up results of less than 1 year (16).

Potential adjunct endoscopic therapies to prevent reflux following POEM include transoral incisionless 
fundoplication (TIF) and radiofrequency ablation to the LES with the Stretta procedure. TIF and Stretta both offer less invasive options to replace surgical fundoplication, have fewer adverse effects and do not limit future treatment options. TIF procedure repairs the anti-reflux barrier through creation of a valve 2 to $4 \mathrm{~cm}$ in length with a 270 degree or greater circumferential wrap endoscopically (17). Stretta utilizes radiofrequency ablation to the LES to create thermal lesions below the mucosa at the gastroesophageal junction and restore a reflux barrier (18). The balance between postoperative GERD and worsening dysphagia must be considered following surgical or endoscopic therapy for achalasia. As POEM becomes a more standardized procedure and providers develop proficiency in this therapy, we may see more patients developing GERD in the postoperative period. The decision to add an antireflux procedure at the time POEM is performed versus selecting patients with symptomatic or diagnostic testing confirming GERD or esophagitis for antireflux procedures later will be a challenge for surgeons and endoscopists. All patients undergoing therapy for achalasia, including PD, LHM and POEM, should be monitored for the development of pathologic GERD. Future studies with longer term follow up for achalasia patients undergoing POEM or SM, as well as studies comparing POEM with and without antireflux procedures will be needed to determine the best therapeutic options for patients. As we move into the future, we may see endoscopic therapies for achalasia and GERD become the standard of care and surgical myotomy become an antiquated procedure we read about in textbooks.

\section{Acknowledgements}

None.

\section{Footnote}

Conflicts of Interest: JM Marks is a consultant for Olympus and Boston Scientific; KF Angell has no conflicts of interest to declare.

\section{References}

1. Vaezi MF, Pandolfino JE, Vela MF. ACG clinical guideline: diagnosis and management of achalasia. Am J Gastroenterol 2013;108:1238-49; quiz 1250.

2. Pandolfino JE, Kahrilas PJ; American Gastroenterological Association. AGA technical review on the clinical use of esophageal manometry. Gastroenterology 2005;128:209-24.

3. Boeckxstaens GE, Annese V, des Varannes SB, et al. Pneumatic dilation versus laparoscopic Heller's myotomy for idiopathic achalasia. N Engl J Med 2011;364:1807-16.

4. Campos GM, Vittinghoff E, Rabl C, et al. Endoscopic and surgical treatments for achalasia: a systematic review and meta-analysis. Ann Surg 2009;249:45-57.

5. Moonen A, Annese V, Belmans A, et al. Long-term results of the European achalasia trial: a multicentre randomised controlled trial comparing pneumatic dilation versus laparoscopic Heller myotomy. Gut 2016;65:732-9.

6. Vela MF, Richter JE, Khandwala F, et al. The long-term efficacy of pneumatic dilatation and Heller myotomy for the treatment of achalasia. Clin Gastroenterol Hepatol 2006;4:580-7.

7. Inoue H, Minami H, Kobayashi $\mathrm{Y}$, et al. Peroral endoscopic myotomy (POEM) for esophageal achalasia. Endoscopy 2010;42:265-71.

8. Swanstrom LL, Kurian A, Dunst CM, et al. Long-term outcomes of an endoscopic myotomy for achalasia: the POEM procedure. Ann Surg 2012;256:659-67.

9. Hungness ES, Teitelbaum EN, Santos BF, et al. Comparison of perioperative outcomes between peroral esophageal myotomy (POEM) and laparoscopic Heller myotomy. J Gastrointest Surg 2013;17:228-35.

10. Teitelbaum EN, Dunst CM, Reavis KM, et al. Clinical outcomes five years after POEM for treatment of primary esophageal motility disorders. Surg Endosc 2017. [Epub ahead of print].

11. Inoue $H$, Sato $H$, Ikeda $H$, et al. Per-Oral Endoscopic Myotomy: A Series of 500 Patients. J Am Coll Surg 2015;221:256-64.

12. Sharata AM, Dunst CM, Pescarus R, et al. Peroral endoscopic myotomy (POEM) for esophageal primary motility disorders: analysis of 100 consecutive patients. J Gastrointest Surg 2015;19:161-70; discussion 170.

13. Shiwaku H, Inoue H, Sasaki T, et al. A prospective analysis of GERD after POEM on anterior myotomy. Surg Endosc 2016;30:2496-504.

14. Rebecchi F, Giaccone C, Farinella E, et al. Randomized controlled trial of laparoscopic Heller myotomy plus Dor fundoplication versus Nissen fundoplication for achalasia: long-term results. Ann Surg 2008;248:1023-30.

15. Rawlings A, Soper NJ, Oelschlager B, et al. Laparoscopic Dor versus Toupet fundoplication following Heller myotomy for achalasia: results of a multicenter, prospective, randomized-controlled trial. Surg Endosc 2012;26:18-26. 
16. Marano L, Pallabazzer G, Solito B, et al. Surgery or Peroral Esophageal Myotomy for Achalasia: A Systematic Review and Meta-Analysis. Medicine (Baltimore) 2016;95:e3001.

17. Jain D, Singhal S. Transoral Incisionless Fundoplication for Refractory Gastroesophageal Reflux Disease: Where

Cite this article as: Angell KF, Marks JM. The future of achalasia therapy: expanding the minimally invasive armamentarium and risk of secondary gastroesophageal reflux. J Thorac Dis 2017;9(10):3659-3662. doi:10.21037/ jtd.2017.09.127
Do We Stand? Clin Endosc 2016;49:147-56.

18. Triadafilopoulos G, DiBaise JK, Nostrant TT, et al. The Stretta procedure for the treatment of GERD: 6 and 12 month follow-up of the U.S. open label trial. Gastrointest Endosc 2002;55:149-56. 